\title{
SNMMI/ABNM Joint Position Statement on Optimizing Training in Nuclear Medicine in the Era of Hybrid Imaging
}

\author{
Dominique Delbeke ${ }^{1}$, Henry D. Royal ${ }^{2}$, Kirk A. Frey $^{3}$, Michael M. Graham $^{4}$, and George M. Segall ${ }^{5,6}$ \\ ${ }^{1}$ Vanderbilt University Medical Center, Nashville, Tennessee; ${ }^{2}$ Mallinckrodt Institute of Radiology, St. Louis, Missouri; ${ }^{3}$ University of \\ Michigan Medical Center, Ann Arbor, Michigan; ${ }^{4}$ University of Iowa, Iowa City, Iowa; ${ }^{5}$ VA Palo Alto Health Care System, Palo Alto, \\ California; and ${ }^{6}$ Stanford University, Stanford, California
}

$\mathbf{T}$ he American Board of Nuclear Medicine (ABNM) was recognized as an independent medical specialty board in 1971 and was the first conjoint board of the American Board of Medical Specialties (ABMS). The conjoint board was sponsored by the American Board of Internal Medicine (therapies), the American Board of Pathology (radioimmunoassay), the American Board of Radiology (ABR) (diagnostic imaging), and the Society of Nuclear Medicine and Molecular Imaging (SNMMI) based on the recommendation of the Liaison Committee for Specialty Boards, the ABMS, and the Council on Medical Education of the American Medical Association. It became a primary medical specialty board whose only sponsor was the SNMMI in 1985. Medical specialties are defined by their core knowledge, not by technologies, because technologies are constantly changing. Nuclear medicine is a primary medical specialty because its core knowledge (exploitation of the tracer principle to study biochemical, physiologic, and molecular processes in time and space) is unique. The ABNM defines nuclear medicine as "the medical specialty that uses the tracer principle, most often with radiopharmaceuticals, to evaluate molecular, metabolic, physiologic and pathologic conditions of the body for the purposes of diagnosis, therapy and research" (1). The ABNM has recently published a statement on nuclear medicine professional competency and scope of practice (2). Nuclear medicine training programs embrace the complete spectrum of the practice of nuclear medicine, including general diagnostic procedures, radionuclide therapies, and cardiac imaging with exercise or pharmacologic agents. Residency programs in nuclear medicine are robust and for the past $40 \mathrm{y}$ have trained most of the nuclear medicine physicians in practice. In 2012-2013, there are 54 Accreditation Council for Graduate Medical Education (ACGME)-accredited nuclear medicine resident training

\footnotetext{
Received Jun. 21, 2012; revision accepted Jun. 22, 2012

For correspondence or reprints contact: Dominique Delbeke, Vanderbilt University Medical Center, 21st Ave. S. and Garland, Nashville, TN 372322675.

E-mail: Dominique.delbeke@vanderbilt.edu

Published online Jul. 26, 2012.

COPYRIGHT (C) 2012 by the Society of Nuclear Medicine and Molecular Imaging, Inc.

DOI: $10.2967 /$ jnumed.112.110346
}

programs, and there are currently 144 on-duty trainees $(3,4)$. ABNM has issued 789 certificates from 2000 to 2012, and the number has remained stable since the early $1980 \mathrm{~s}$ (Fig. 1).

The ABR began offering its own certificate of competence in nuclear radiology in 1973. Nuclear radiology was established as a subset of nuclear medicine limited to nuclear diagnostic imaging. Fellowship programs in nuclear radiology have been created that lead to eligibility for subspecialty certification in nuclear radiology by the ABR. Physicians must have ABR certification in diagnostic radiology and have an additional year of fellowship training in nuclear radiology in one of the 19 ACGME-accredited nuclear radiology training programs. In 2012-2013, there are 18 onduty trainees $(3,4)$. This training pathway includes a total of 16 mo in nuclear radiology: 4 mo during diagnostic radiology residency and 12 mo during a nuclear radiology fellowship. In 2011, the ABR created a second pathway for subspecialty certification in nuclear radiology consisting of 16 mo of training in nuclear radiology or nuclear medicine during $4 \mathrm{y}$ of diagnostic radiology residency, of which 10 mo must be consecutive. A significant difference between nuclear medicine and nuclear radiology is the amount of training required for therapy with radiopharmaceuticals as described in a conjoint statement on credentialing and delineation of privileges for therapeutic procedures using radiopharmaceuticals (5).

\section{Hybrid Imaging and Molecular Imaging}

The emergence of hybrid imaging in nuclear medicine has changed the scope of knowledge required for nuclear medicine physicians. Nuclear medicine physicians must now have a greater knowledge of 3-dimensional anatomy. In addition, molecular imaging is an evolving field with the development of new targeted radiopharmaceuticals and other tracers that will allow identification of patients with specific characteristics who will benefit from targeted treatments. However, not all nuclear medicine procedures are molecular imaging procedures. For example, perfusion imaging of the lungs or gated blood pool imaging of the heart are not molecular procedures because blood flow is a physiologic, not a molecular, process.

In 2005, a molecular imaging summit sponsored by the Radiologic Society of North America and the SNMMI 


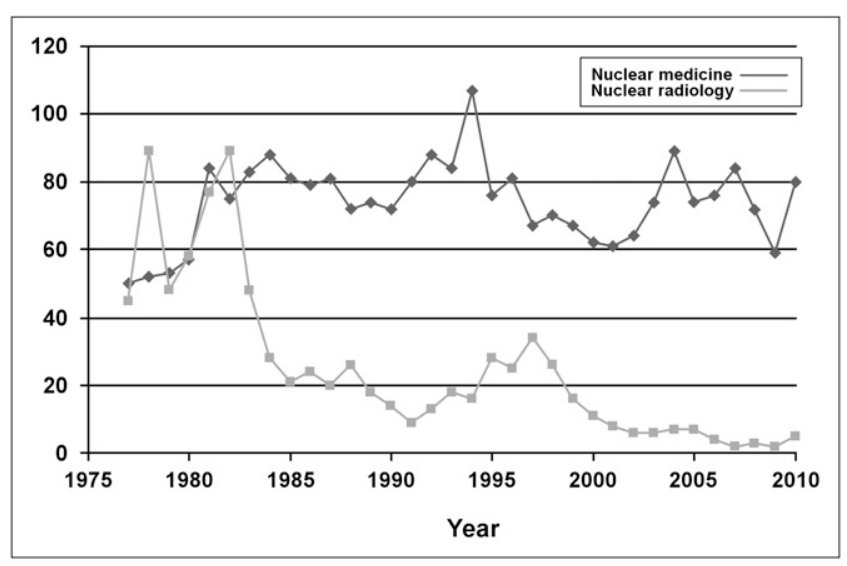

FIGURE 1. Number of certificates granted by ABNM and ABR for subspecialty in nuclear radiology.

recommended the following definition (6): "Molecular imaging techniques directly or indirectly monitor and record the spatiotemporal distribution of molecular or cellular processes for biochemical, biologic, diagnostic, or therapeutic applications." In 2007, the SNMMI adopted the following definition of molecular imaging (7): "Molecular imaging is the visualization, characterization, and measurement of biological processes at the molecular and cellular levels in humans and other living systems. Molecular imaging typically includes two- or three-dimensional imaging as well as quantification over time. There are several imaging modalities that can be used for [molecular imaging], including Positron Emission Tomography (PET), Single Photon Emission Computed Tomography (SPECT), Magnetic Resonance Imaging (MRI), Ultrasound (US), Optical Imaging (OI), and potentially others. Molecular imaging agents are tracers used to visualize, characterize, and measure biologic processes in living systems. Both endogenous molecules and exogenous probes can be molecular imaging agents." At the present time, virtually all molecular imaging procedures that have clinical applications and are available for patient care use radioactive tracers. In the future, nonradioactive tracers will likely become available for clinical use.

To optimize the evolution of molecular imaging, it is likely that the tracer principle will be applied clinically using other technologies (ultrasound, magnetic resonance, optical imaging), and it will therefore be important to train residents and the nuclear medicine workforce in the use of these new technologies. Because each of these new technologies has limitations in its ability to detect tracers, it is still unclear how soon and how widespread their clinical impact will be.

These issues are also being recognized worldwide and have been addressed by the European Association of Nuclear Medicine $(8-11)$.

\section{Existing Traditional Nuclear Medicine Residency Training Pathways}

The SNMMI and ABNM have recognized the importance of requiring more training in anatomic imaging for nuclear medicine residents. In July 2007, primary nuclear medicine residency training was lengthened from $2 \mathrm{y}$ to $3 \mathrm{y}$ to accommodate more training in anatomic imaging. Six months of training in cross-sectional anatomic imaging are now required. Several alternative nuclear medicine training pathways exist: $2 \mathrm{y}$ of nuclear medicine if a physician is eligible for certification by another ABMS specialty board; $1 \mathrm{y}$ of nuclear medicine after satisfactorily completing $4 \mathrm{y}$ of training in diagnostic radiology; or 16 mo of nuclear medicine for residents enrolled in a 4-y diagnostic radiology residency training program, 12 mo of which need to be in an ACGME-accredited nuclear medicine program (4 y combined diagnostic radiology and nuclear medicine program). These pathways are described in more detail in Table 1.

\section{SNMMI/ABNM Recommendations for Combined Training in Diagnostic Radiology and Nuclear Medicine}

In 2009, the American College of Radiology-SNMMI Task Force on Nuclear Medicine Training was convened to propose methods to optimize nuclear medicine training. The report was published simultaneously in the June issues of The Journal of Nuclear Medicine and the Journal of the American College of Radiology (12) and summarized the multiple training pathways that currently exist. These pathways can lead to certification by 2 different ABMS member boards: the ABNM, and the ABR with subspecialization in nuclear radiology.

The task force made several recommendations on future training (12). Recommendations for the short-term horizon were to harmonize nuclear medicine residency and nuclear radiology fellowship program requirements, improve the robustness of nuclear medicine training in diagnostic radiology residencies, improve the robustness of diagnostic radiology training in nuclear medicine residencies, and encourage a 16-mo nuclear medicine pathway within diagnostic radiology residencies. For the long-term horizon, the recommendation was to develop combined diagnostic radiology and nuclear medicine training programs for molecular imaging specialists.

Regarding the first recommendation, SNMMI, ABNM, and the nuclear medicine residency review committee (RRC) believe that nuclear medicine residency and nuclear radiology fellowship programs should be unified under a single expanded nuclear medicine RRC including representatives of radiology organizations, whereas the diagnostic radiology RRC has proposed having nearly identical but separate programs, one under the jurisdiction of the diagnostic radiology RRC and one under the jurisdiction of the nuclear medicine RRC. Having separate but equal subspecialty training programs will lead to competition rather than collaboration and is inefficient, especially given the small numbers of trainees. Such a system is also inconsistent with a 40-y-old agreement in which nuclear radiology was established as a subset of nuclear medicine limited to nuclear diagnostic imaging, not the full practice of 


\begin{tabular}{|c|c|c|}
\hline $\begin{array}{l}\text { Duration of nuclear } \\
\text { medicine training }\end{array}$ & Requirements & Certification \\
\hline $4 \mathrm{mo}$ & Diagnostic radiology residency program & ABR (diagnostic radiology) \\
\hline \multirow[t]{2}{*}{$16 \mathrm{mo}$} & $\begin{array}{l}4 \text { y diagnostic radiology (including } 4 \text { mo nuclear medicine) + } \\
1 \text { y nuclear medicine }\end{array}$ & ABNM or $\mathrm{ABR}$ (nuclear radiology) \\
\hline & 4 y diagnostic radiology (including 16 mo nuclear medicine) & ABNM or ABR (nuclear radiology) \\
\hline $24 \mathrm{mo}$ & $\begin{array}{l}2 \text { y nuclear medicine (including } 6 \text { mo CT) if board-eligible by } \\
\text { another ABMS specialty board }\end{array}$ & ABNM \\
\hline $36 \mathrm{mo}$ & 3 y nuclear medicine (including 6 mo CT) after 1 y clinical & ABNM \\
\hline
\end{tabular}

nuclear medicine (13). The task force made no proposals to improve the robustness of nuclear medicine training in diagnostic radiology residencies or to improve the robustness of diagnostic radiology training in nuclear medicine residencies. In 2005, diagnostic radiology training programs decreased the duration of required training in nuclear medicine from 6 mo to 4 mo (16 wk), subsequent to a change in Nuclear Regulatory Commission regulations regarding authorized users of radioactive materials. In contrast, nuclear medicine training programs increased the length of training from $2 \mathrm{y}$ to $3 \mathrm{y}$ in 2007 to accommodate more training in cross-sectional imaging. In a letter dated October 28, 2009, the ABR objected to the proposed changes because the $\mathrm{CT}$ training required was separate from SPECT/CT or PET/CT, imposed a burden on diagnostic radiology programs, and was "woefully inadequate." Arbitration facilitated by the ACGME resulted in the inclusion of "participate in a minimum of six months of CT experience; a minimum of four months must be obtained on a [diagnostic radiology] CT service."

The American College of Radiology has proposed a 4-y and a 5-y pathway for combined training in diagnostic radiology and nuclear medicine with global oversight by the diagnostic radiology RRC (14). The 4-y combined diagnostic radiology/nuclear medicine training pathway is now possible because diagnostic radiology program requirements (effective July 2010) permit 16 mo of training in any one subspecialty, including nuclear medicine. If at least 12 mo of the 16 mo of nuclear medicine training are done in an accredited nuclear medicine training program, these residents will meet the requirements of both the ABR and the ABNM. The 5-y pathway would consist of $3 \mathrm{y}$ of training in diagnostic radiology and 2 additional years of training in nuclear medicine (14). A 5-y combined diagnostic radiology/nuclear medicine training program is important for trainees who want more comprehensive training in nuclear medicine with more research opportunities.

Having a collaborative rather than competitive relationship with diagnostic radiology will optimize the future of nuclear medicine and molecular imaging. Oversight of nuclear medicine training should be done by a single expanded nuclear medicine RRC, which can focus on the core knowledge and issues facing the primary specialty of nuclear medicine. This focused oversight will be especially important for advanced training that includes more scholarly projects for trainees, and role modeling of faculty for future imager-scientists. Most nuclear medicine training has been under the guidance of nuclear medicine professionals for the past $40 \mathrm{y}$. Leaders in nuclear medicine have led research and progress in nuclear medicine. The large number of nuclear medicine residency programs, the long track records of experience of nuclear medicine professionals, and their expertise with the entire scope of nuclear medicine procedures makes an expanded nuclear medicine RRC a natural choice to have oversight over nuclear medicine training in the future. However, despite the need for imagers with combined diagnostic radiology and nuclear medicine training, there will still be a need for physicians trained in a single specialty who practice a limited scope of nuclear medicine (mostly diagnostic radiologists) or have a highly focused practice (mostly nuclear medicine research).

The ABNM is facilitating the establishment of combined diagnostic radiology and nuclear medicine training programs by holding frequent conference calls with interested nuclear medicine program directors to help them solve some of the administrative problems related to the implementation of a combined program. Implementation of these combined programs has been slowed by disagreements about the supervising RRC.

\section{ACGME and National Resident Match Program: Combined Residency Training}

Combined programs are addressed on the ACGME Web site (15) (see "Programs with Combined Specialty Tracks" in the left-menu search function). According to ACGME, "Combined training consists of a coherent educational experience in two or more closely related specialties or subspecialties available for selected individuals." The educational plan for combined training is approved by the specialty board of each of the specialties to ensure that resident physicians completing combined training are eligible for board certification in each of the component specialties. Each specialty or subspecialty program is separately accredited by ACGME through its respective specialty review committee. The ACGME is no longer considering formal accreditation of combined residency programs as a single 
program. However, ACGME will assign a program number that allows institutions to enroll residents in combined programs through the National Resident Match Program.

\section{SNMMI and ABNM Recommendations for Stratified Levels of Training and Experience in Nuclear Medicine}

Because training in nuclear medicine can vary from 4 mo during a diagnostic radiology residency program to $3 \mathrm{y}$ in a nuclear medicine residency program, the SNMMI and ABNM recommend the adoption of stratified levels of training with competency explicitly linked to duration and content of training. The levels-of-training approach has been described and implemented in cardiac imaging during the Core Cardiology Training Symposium and was last revised in 2006 for nuclear cardiology (16). Training of residents and fellows in nuclear cardiology is divided into 3 levels of competency and expertise: general competency (level 1, minimum of $2 \mathrm{mo}$ ) makes trainees acquainted with the field, specialized competency (level 2, minimum of 4 mo) provides trainees with expertise to practice the limited field of nuclear cardiology, and advanced competency (level 3, minimum of $12 \mathrm{mo}$ ) provides training sufficient to be the director of an imaging laboratory or to pursue an academic career.

This stratified approach has also been suggested for nuclear medicine training and experience by the External Stakeholders Working Group of the SNMMI 2020 Task Force appointed early in 2011. The purpose of the SNMMI 2020 task force was to bring together a broad cross-section of health care professionals to discuss the future of the nuclear medicine profession and make recommendations that will serve as guiding principles to meet the challenges for the profession and the field. The external stakeholders group included leaders in radiology and cardiology. The SNMMI 2020 task force working groups met on September 16, 2011, in McLean, Virginia, to present their reports. One of the recommendations of the external stakeholders working group was to consider designated levels of nuclear medicine clinical practice, such as the 3 levels in nuclear cardiology. Level 1 would indicate knowledge of the field and an ability to recommend tests and interpret certain common procedures. Level 2 would signify a practitioner able to recommend, perform, and interpret a broad range of nuclear medicine studies. Level 3 would be reserved for trainees with knowledge and experience to serve as an imaging and therapeutic laboratory director. With teleradiology, a level 1 practitioner could practice under the supervision of a level 2 or level 3 practitioner, who could be in another location.

The external stakeholders group also recommended defining competency in organ systems and in multimodality/hybrid imaging (Guiberteau M, et al., written communication, September 16, 2011). Defined levels of competency would promote excellence in clinical practice and improve patient safety. Having levels of practice linked to the intensity and duration of training would be a much better system than the current system in which trainees with only 4 mo of nuclear medicine training are considered to be "imminently (sic) qualified for the full practice of nuclear medicine" (17) and are not distinguished from those with more extensive training experience. The concept of levels of training is new for nuclear medicine, and the appropriate length and scope for each level of training should be defined with all stakeholders in the future.

\section{Continuing Education and Maintenance of Certification}

Technology changes rapidly, and medical knowledge continues to increase. Because of these rapid changes, physicians must be committed to a lifetime of learning, and there must be a credible system in place to document that physicians have kept up with advances in their specialty. Maintenance of certification, which was implemented by the ABNM in 2007, provides a framework to document that working physicians are keeping up to date with their specialty as new technologies are introduced. Participation in maintenance of certification will become increasingly critical in the future. Educational endeavors that focus solely on resident training will not be effective in changing the capabilities of the workforce.

\section{SUMMARY}

In the future, nuclear medicine training will expand to include more training in anatomic and molecular imaging, including nonradioactive imaging modalities using the tracer principle.

The SNMMI and ABNM believe that...

1. A single expanded nuclear medicine RRC, including representatives of nuclear medicine and radiology organizations, should have oversight of nuclear medicine training under local nuclear medicine program directors.

2. Physicians practicing nuclear medicine will benefit from combined training in diagnostic radiology and nuclear medicine, with subsequent improvements to patient care and advancement of the field.

3. Four-year and 5-y combined diagnostic radiology and nuclear medicine pathways should be supported.

4. Existing training pathways in nuclear medicine will be needed in the future to train physicians practicing in a broad variety of clinical and academic settings.

5. Stratified levels of training and competency in nuclear medicine should be adopted to promote quality and safety.

6. Participation in maintenance of certification is critical to maintain competence with rapidly evolving technologies.

\section{REFERENCES}

1. Home page. The American Board of Nuclear Medicine Web site. Available at: http://www.abnm.org. Accessed June 29, 2012. 
2. Frey KA, Royal HD, Di Carli MF, et al. ABNM position statement: nuclear medicine professional competency and scope of practice. J Nucl Med. 2011;52:994-997.

3. Number of accredited programs by academic year. The Accreditation Council for Graduate Medical Education Web site. Available at: http://www.acgme.org/adspublic/ reports/accredited_programs.asp. Accessed June 29, 2012.

4. Delbeke D, Segall GM. Status of and trends in nuclear medicine in the United States. J Nucl Med. 2011;52(suppl):24S-28S.

5. Delbeke D, Graham MG, Royal H, Seagall G, Shulkin BL, Ziessman HA. Conjoint statement of the SNM, ACNM and ABNM on credentialing and delineation of privileges for therapeutic procedures using radiopharmaceuticals. J Nucl Med. 2011;52:323-326.

6. Thakur ML, Lentle BC. Joint SNM/RSNA molecular imaging summit statement. J Nucl Med. 2005;46(9):11N-13N, 42N.

7. Mankoff D. A definition of molecular imaging. J Nucl Med. 2007;48(6):18N, $21 \mathrm{~N}$.

8. Bischof Delaloye A, Carrio I, Cuocolo A, et al. White paper of the European Association of Nuclear Medicine (EANM) and the European Society of Radiology (ESR). Eur J Nucl Med Mol Imaging. 2007;34:1147-1151.

9. Cuocolo A, Breatnach E. Multimodality imaging in Europe: a survey by the European Association of Nuclear Medicine (EANM) and the European Society of Radiology (ESR). Eur J Nucl Med Mol Imaging. 2010;37:163-167.

10. European Association of Nuclear Medicine (EANM). European Society of Radiology (ESR): Multimodality imaging training curriculum—general recommendations. Eur J Nucl Med Mol Imaging. 2011;38:976-978.
11. European Association of Nuclear Medicine (EANM); European Society of Radiology (ESR). Multimodality imaging training curriculum: parts II and III. Eur J Nucl Med Mol Imaging. 2012;39:557-562.

12. Guiberteau MJ, Graham MM. ACR-SNM task force on nuclear medicine training: report of the task force. J Nucl Med. 2011;52:998-1002.

13. Linton OW. The American Board of Radiology: 75 Years of Serving the Public. Tucson, AZ: American Board of Radiology; 2009: 95-113.

14. Oates ME; Diagnostic Radiology Participants of ACR/SNM Task Force II. Integrated residency training pathways of the future: diagnostic radiology, nuclear radiology, nuclear medicine and molecular imaging. J Am Coll Radiol. 2012; 9:239-244.

15. List of ACGME Accredited Programs and Sponsoring Institutions. The Accreditation Council for Graduate Medical Education Web site. Available at: http:// www.acgme.org/adspublic/. Accessed June 29, 2014.

16. Cerquiera MD, Berman DS, Di Carli MF, Schelbert HR, Wackers FJ, Williams KA. Task force 5: training in nuclear cardiology-endorsed by the American Society of Nuclear Cardiology. J Am Coll Cardiol. 2006;47:879904.

17. ACR/ABR writing group. ACR/ABR clinical statement on credentialing and privileging of radiologists for diagnostic nuclear medicine, including multimodality hybrid imaging. J Am Coll Radiol. 2011;8:617-621. 